\title{
Effectiveness of Acceptance and Commitment Therapy on Sexual Self-Esteem, Emotional Skillfulness and Marital Adjustment in Veterans' Wives
}

\section{ART ICLE INF O}

\section{Article Type}

Original Research

\section{Authors}

Pirani Z.*PhD,

Abbasi M. ${ }^{1} P h D$,

Kalvani M. ${ }^{1} M S c$,

Nourbakhsh P. ${ }^{2} M S c$

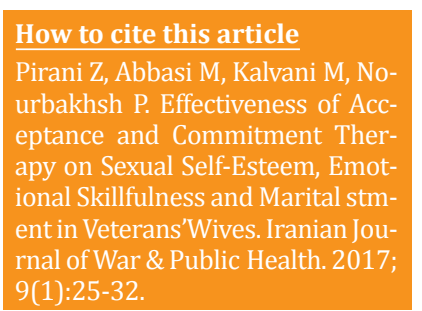

*Psychology Department, Human Science Faculty, Arak Branch, Islamic Azad University, Arak, Iran

${ }^{1}$ Psychology Department, Literature \& Human Science Faculty, Salman Farsi University of Kazerun, Kazerun, Iran

${ }^{2}$ Psychology Department, Human Science Faculty, Arak Branch, Islamic Azad University, Arak, Iran

\section{Correspondence}

Address: Islamic Azad University, Amir Kabir University Town, Kilometer 3 of Khomein Road, Imam Khomeini Square, Arak, Iran Phone: +98 (45) 32721517 Fax: $+98(721) 2229080$ zabih_pirani@yahoo.com

\section{Article History}

Received: October 27, 2016

Accepted: December 12, 2016

ePublished: March 10, 2017

\section{A B S T R A C T}

Aims Due to many marital psychological problems and many stressful mental conditions, the quality of life of the veterans' spouses decreases. The aim of the study was to investigate the effects of acceptance and commitment therapy (ACT) on the sexual self-esteem, alexithymia, and marital adjustment in the veterans' spouses.

Materials \& Methods In the controlled pretest-posttest semi-experimental applied study, 40 veterans' spouses of Arak were studied in 2015. The subjects, selected via available sampling method, were randomly divided into two groups including experimental and control groups ( $n=20$ persons per group). Data was collected using women's sexual self-esteem index (SSELW-SF), Locke-Wallace marital adjustment, and Toronto alexithymia scale (TAS-20). Ten ACT sessions were conducted in experimental group, while control group received no intervention. Pretest and posttest were conducted in both groups. Data was analyzed by SPSS 23 software using descriptive statistics and one-variable covariance analysis test.

Findings After the intervention, the mean scores of sexual self-esteem and marital adjustment significantly increased, while the mean score of alexithymia significantly decreased in experimental group compared to control group $(\mathrm{p}<0.001)$.

Conclusion The ACT increases sexual self-esteem and marital adjustment, while reduces the alexithymia in the veterans' spouses.

Keywords Acceptance and Commitment Therapy; Self-Esteem; Alexithymia; Veterans; Spouses

\section{I T A T I O N L I N K S}

[1] The effect of counseling on veteran's family ... [2] Comparing between psychological health ... [3] Study of some mental disorders in children of fatherless... [4] The relationship between depression and how veterans ... [5] Secondary traumatization and self-rated health among wives ... [6] Marital adjustment parental functioning and emotional ... [7] The relationship of body image and self-esteem with marital ... [8] The Effectiveness of enhancement program ... [9] Perceived criticism and marital adjustment predict ... [10] Marital adjustment and space ... [11] Factors of emotional intelligence associated ... [12] Emotional skillfulness in African marriage ... [13] Emotional skill and marital health... [14] Emotional skillfulness in marriage; Intimacy ... [15] Emotion skills, problem-solving, and marital ... [16] The structure of ... [17] Promotion emotional expression and emotion ... [18] Emotional intelligence and martial adjustment of married ... [19] The effect of psychologicalstatus of students... [20] Self-esteem, self-efficay and self-directedlearning: Attempting ... [21] Psychopathy, sexual behavior and self-esteem ... [22] The correlation between self-esteem and sexual ... [23] Role of sexual satisfaction, spiritual health ... [24] The effectiveness of acceptance and commitment ... [25] A practical guide to acceptance and commitment ... [26] Mindfulness meditation improves cognition ... [27] The effects of mindfulness-based stress ... [28] Effects of mindfulness based stress ... [29] Psychometric properties of persian version ... [30] The effectiveness of acceptance and commitment ... [31] Effectiveness of acceptance and commitment ... [32] Effectiveness of acceptance and commitment ... [33] The role of personal values, acceptance ... [34] The Efficacy of acceptance and commitment ... [35] Mindfulnessbased relationship ... [36] Effectiveness of acceptance and commitment ... [37] Meaning-making intervention during ... [38] Theoretical and practical research ... [39] Reliability and validity of the sexual ... [40] Toronto alexithymia scale: Questionnaire ... [41] New directions in cognitive behavior ... [42] Acceptance and commitment ... [43] Structural model of the relationship ... [4.4] Acceptance and commitment therapy ... [45] Comparison of experimental ... [46] Effect of group acceptance and commitment ... 
مىشوند[3] و اين امر واقعنكرى را در مورد مشكلات جانبازان

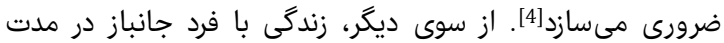

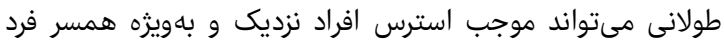

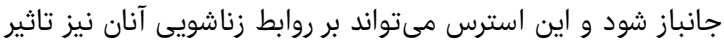

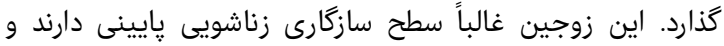

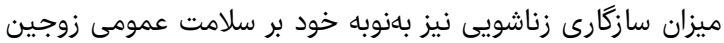

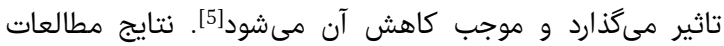

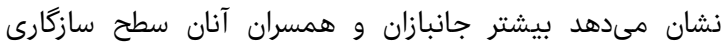

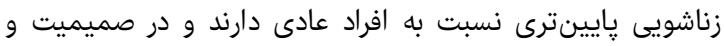

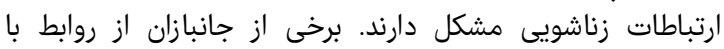

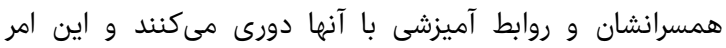

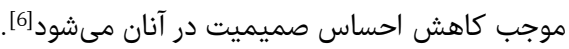

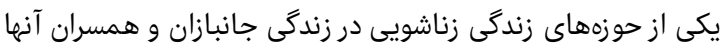

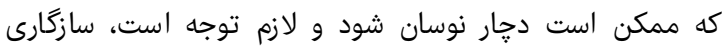

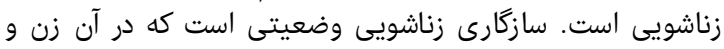

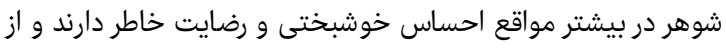

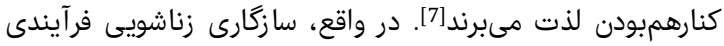

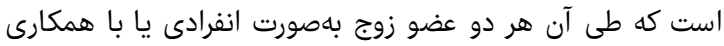

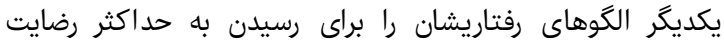

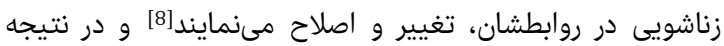

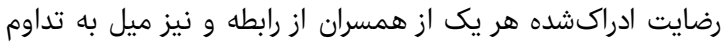

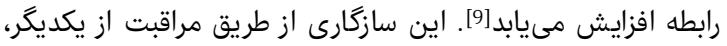

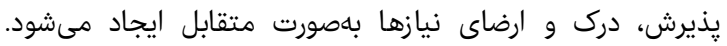

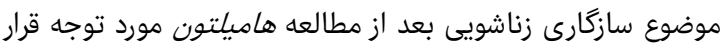

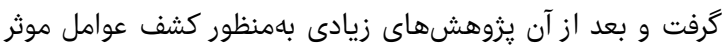

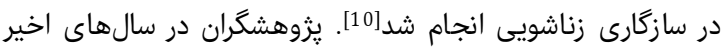

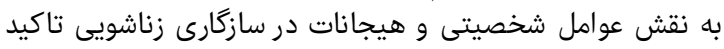

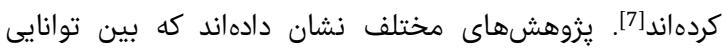

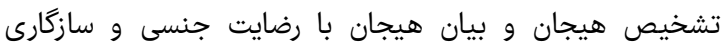
زناشويى[11] و صميميت[ن]

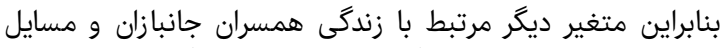

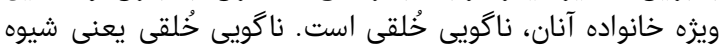

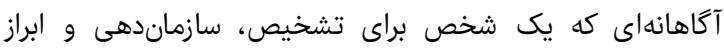

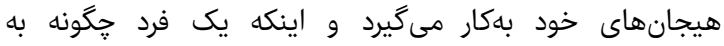

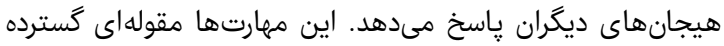

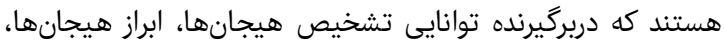

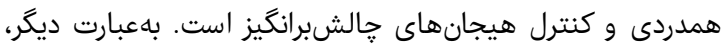

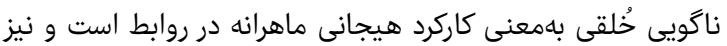

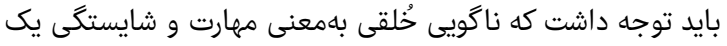

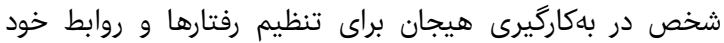

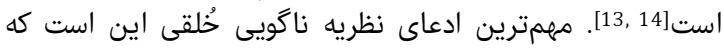

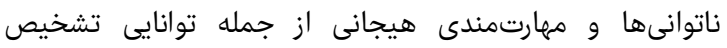

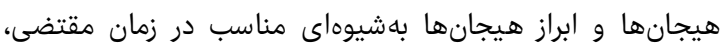

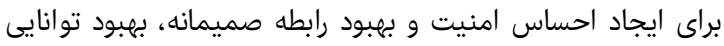

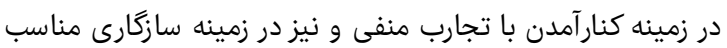

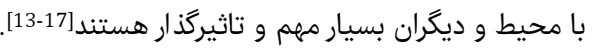

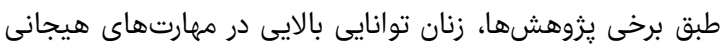

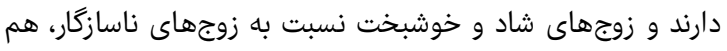

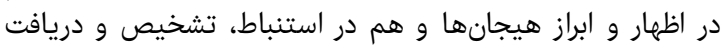

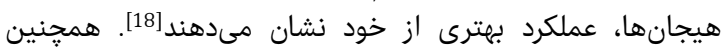

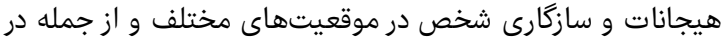
موقعيت زناشويى همسران جانبازان مىتواند متاثر اندان از عزت نفس

دوره ، شماره ا، زمستان هوس"
تاثير درمان مبتنى بر يذيرش و تعهد بر عزت نفس

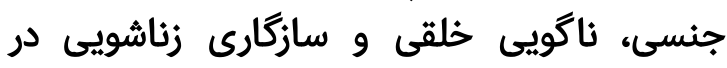

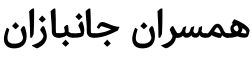

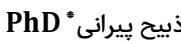

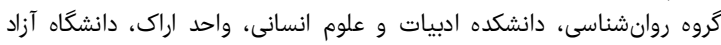
اسلامى، اراك، ايران

PhD مسلم عباسى أراس، ايران

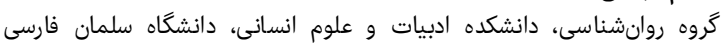
كازرون، كازرون، ايران

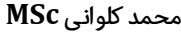
كرّه روانشناسى، دانشكده ادبيات و وعلوم انسانى، دانشكاه سلمان فارسى

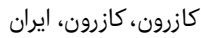

MSc بريناز نوربخش، كازرون ايران

كَروه روانشناسى، دانشكده ادبيات و علوم انسانى، واحد اراك، دانشگاه آزاد

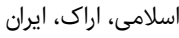

جكيده

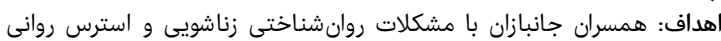

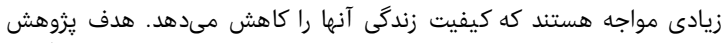

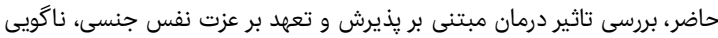

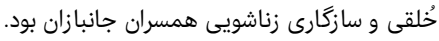

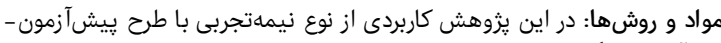

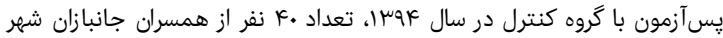

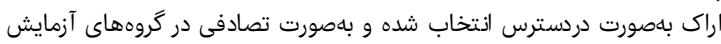

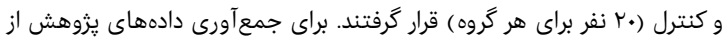

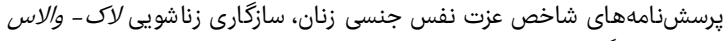

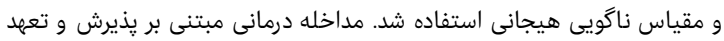

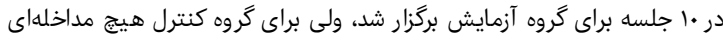

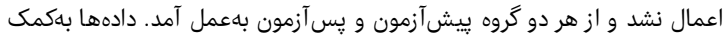

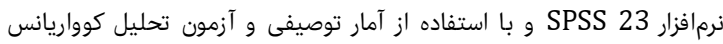

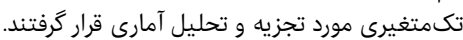

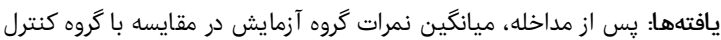

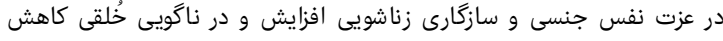

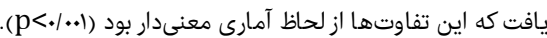

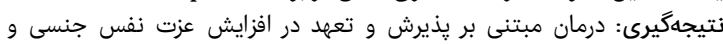

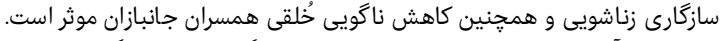

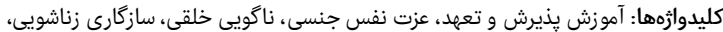

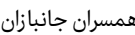

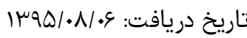

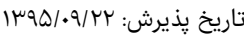

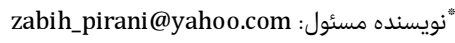

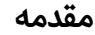

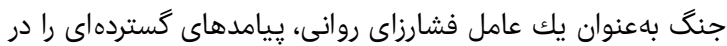

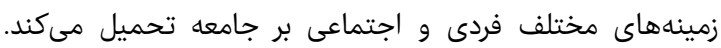

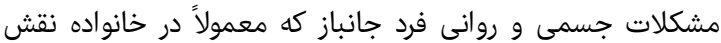

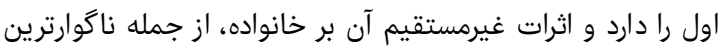

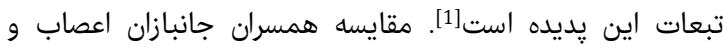

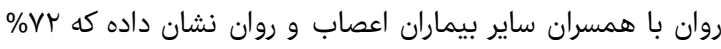

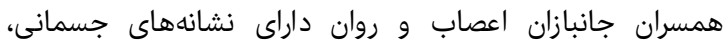

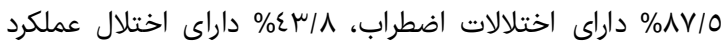

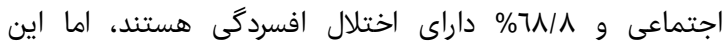

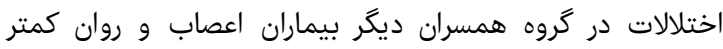

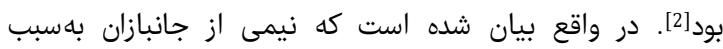

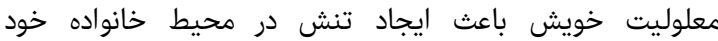

فصل نامه علمى - يخروهشى طب جانباز 


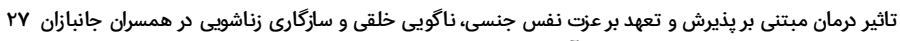

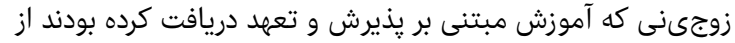

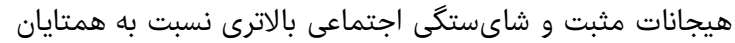
خود در گروه كنترل برخوردار بودند [37].

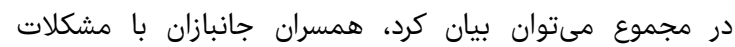

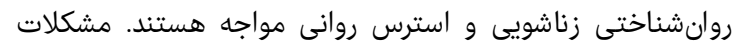

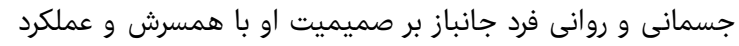

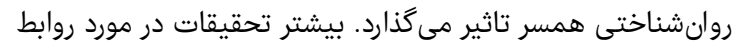

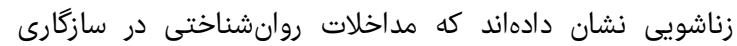

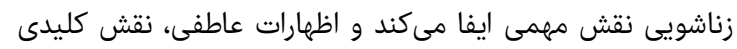

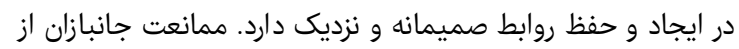

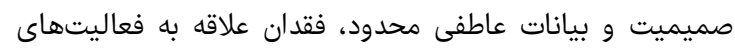

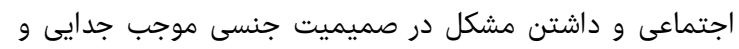

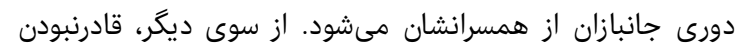

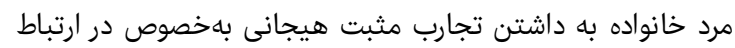

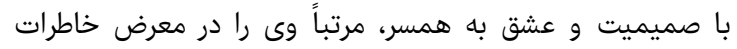

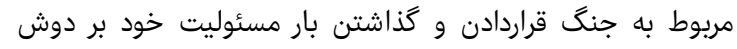

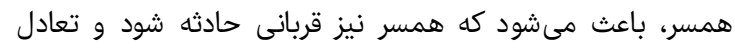

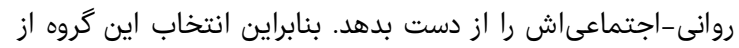

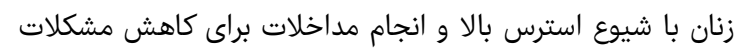

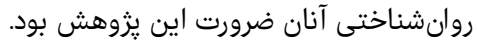

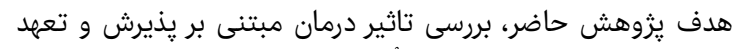

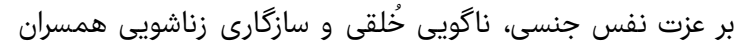

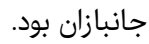

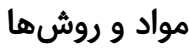

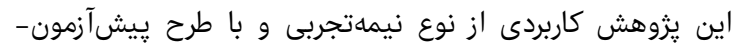

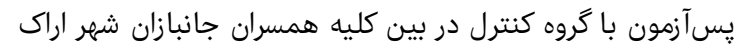

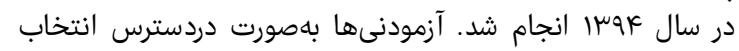

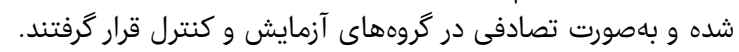

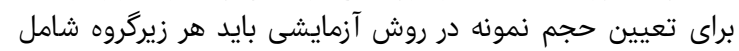

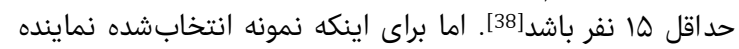

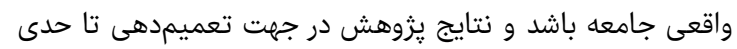

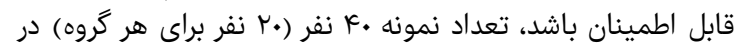

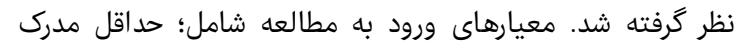

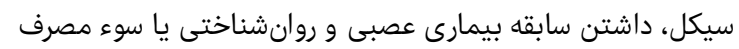

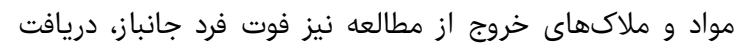

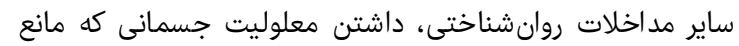

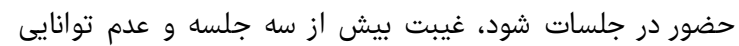

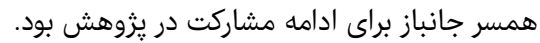

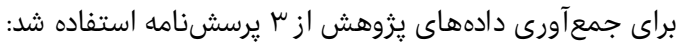

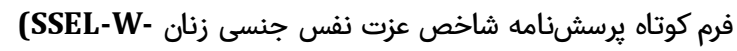

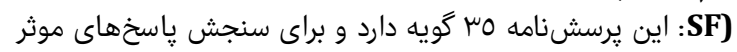

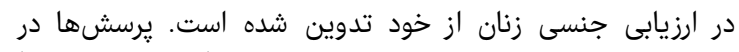

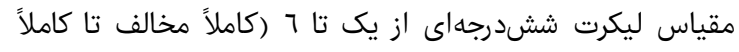

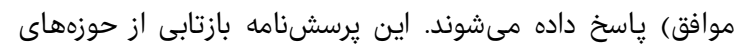

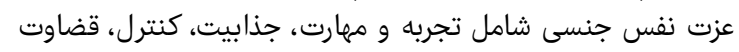

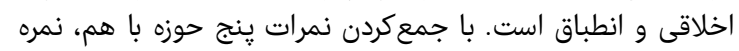

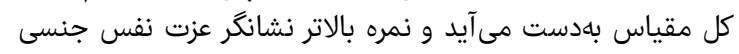

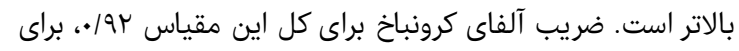

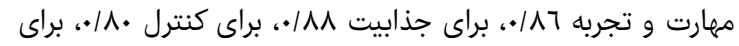

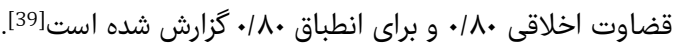

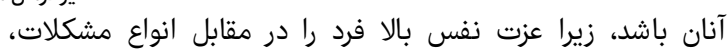

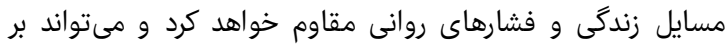

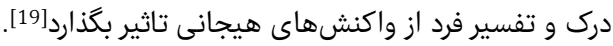

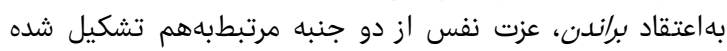

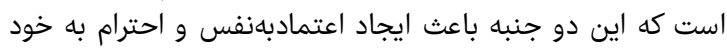

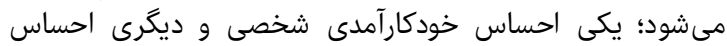

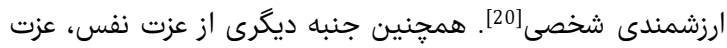

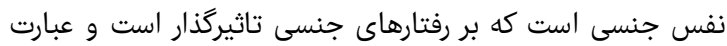

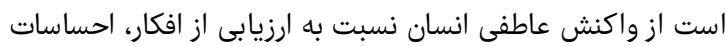

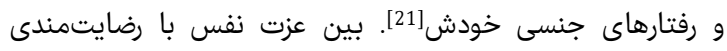

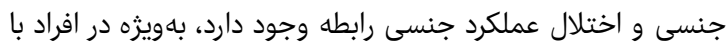

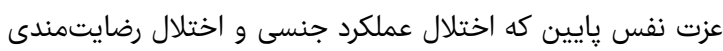

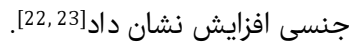

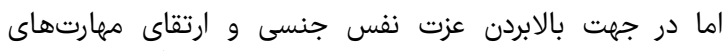

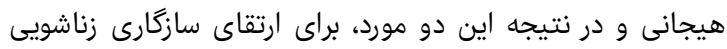

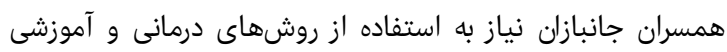

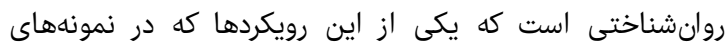

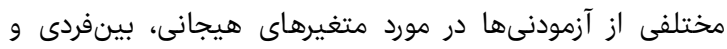

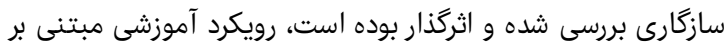

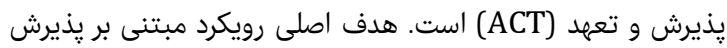

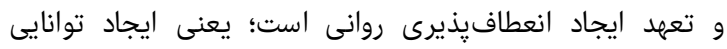

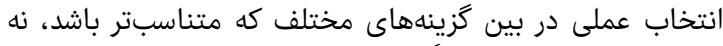

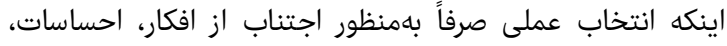

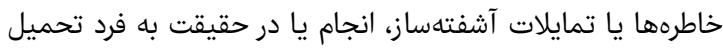

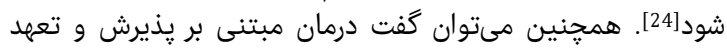

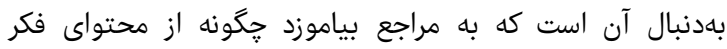

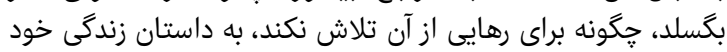

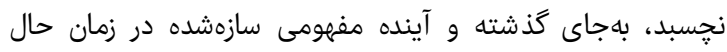

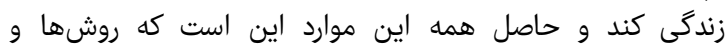

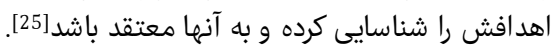

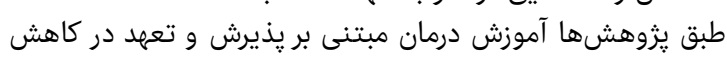

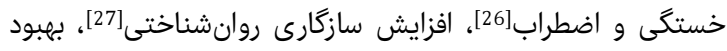

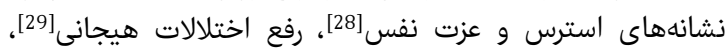

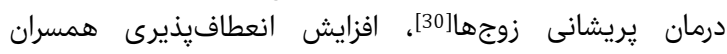

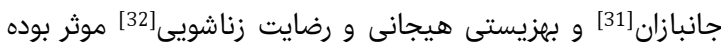

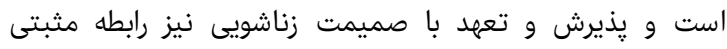

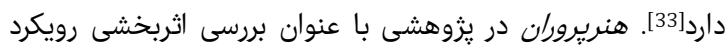

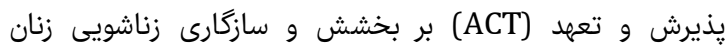

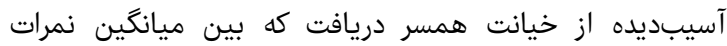

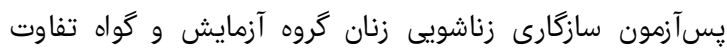

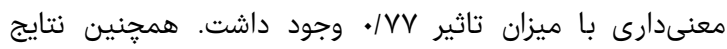

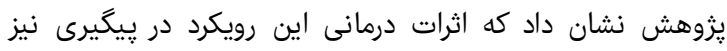

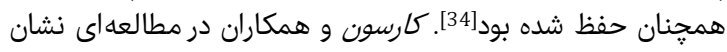

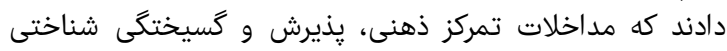

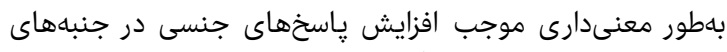

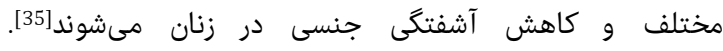

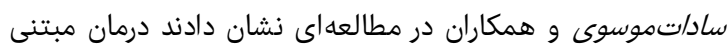

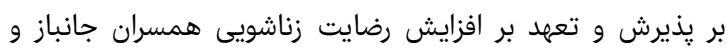

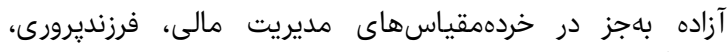

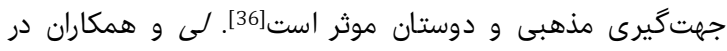

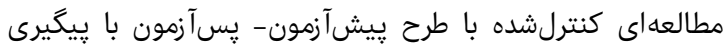

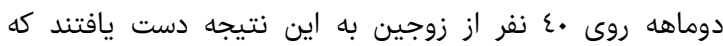




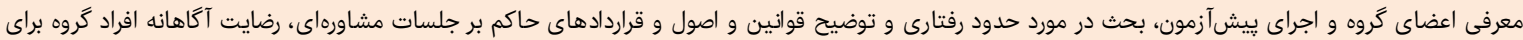

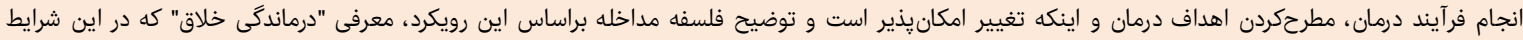

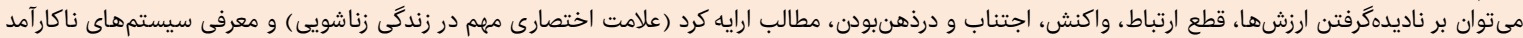

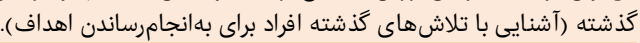

جلسه دوم آنائر

آشنايى با مفاهيم درمانى ACT (انعطافيذيرى روانى، يذيرش روانى، آكاهى روانى، جداسازى شناختى، خودتجسمى، داستان شخصى، روشنسازى ارزشها و عمل متعهدانه ) در شش مرحله:

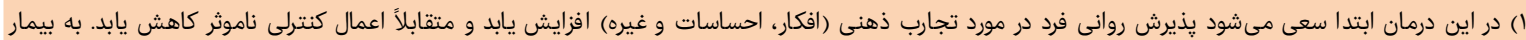

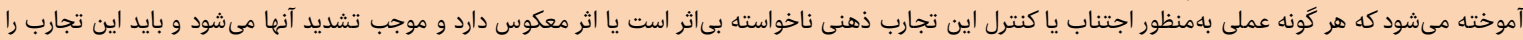

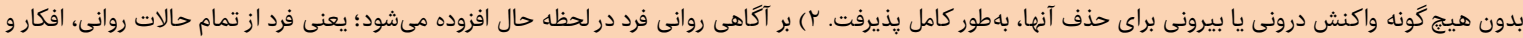

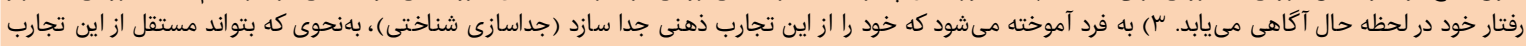

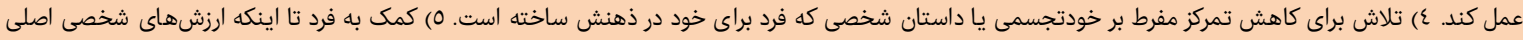

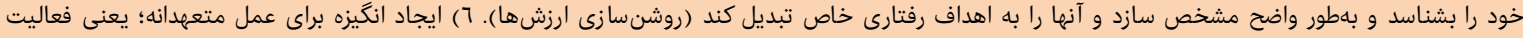

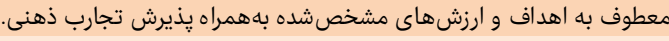

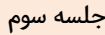

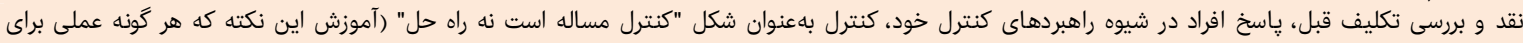

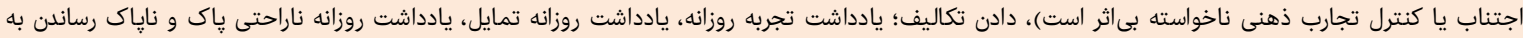
ناميدى خلاق. جلسه جهبارم

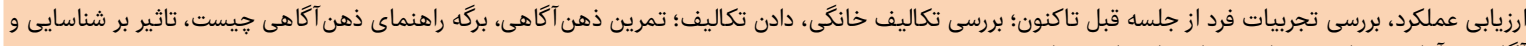

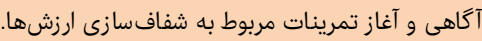

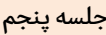

ارزيابى عملكرد، تمايز خودمفهومسازى در برابر خودمشاهدهرىى، سنجش توانايى درمانجو براى گسست از افكار و احساسات، ادامه تجربيات ذهنآكاهى و ادامه شفافسازى ارزشها.

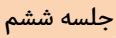

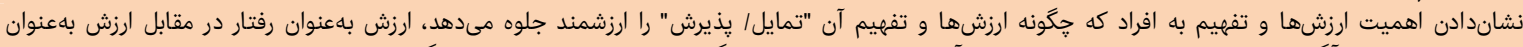

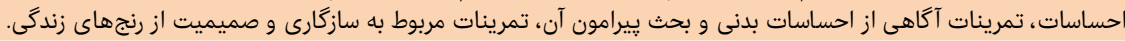
جلسه هفتم

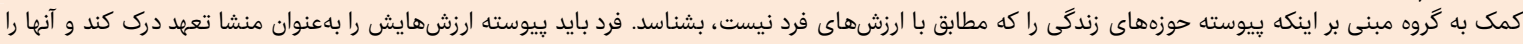

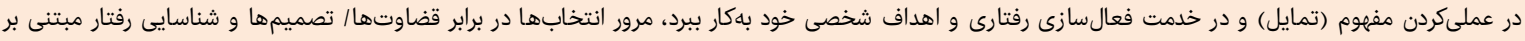

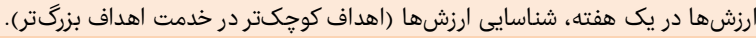

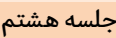

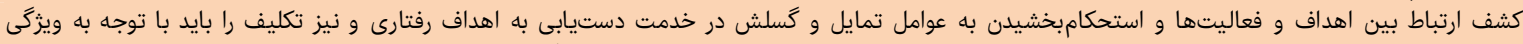

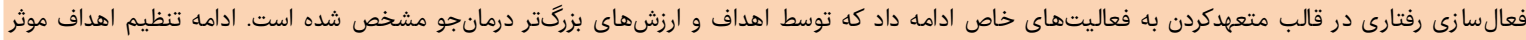

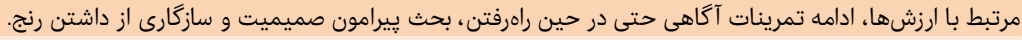

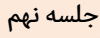

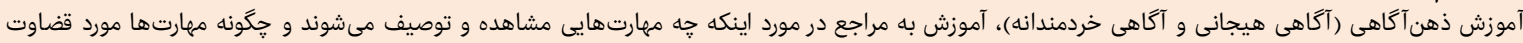

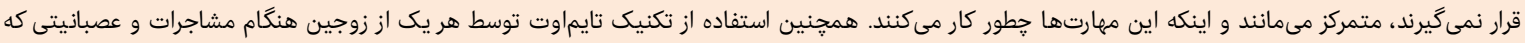

منجر به اختلاف شود.

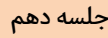

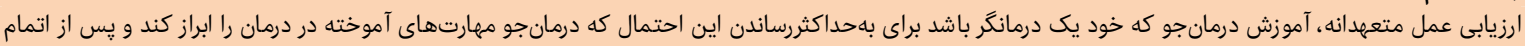

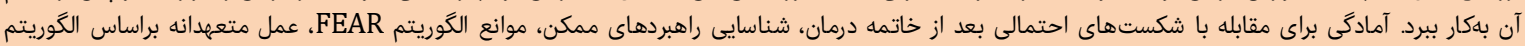
و و ييام اصلى ACT

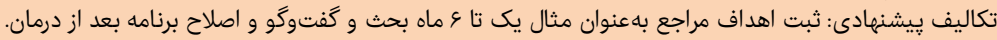

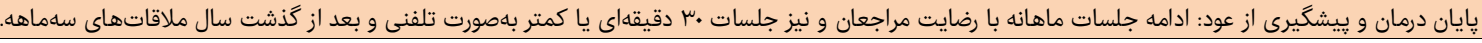

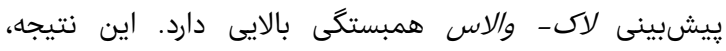

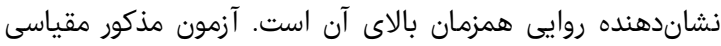

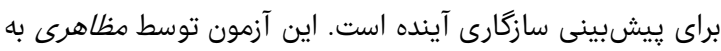

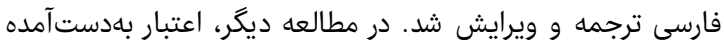

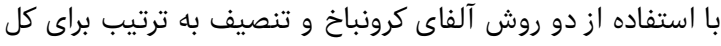

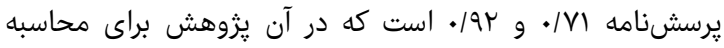

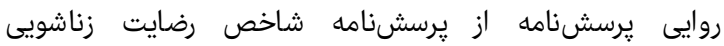

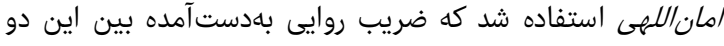

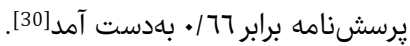

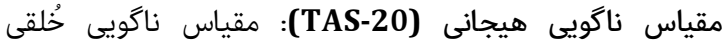

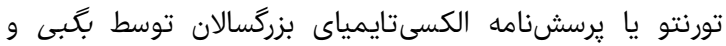

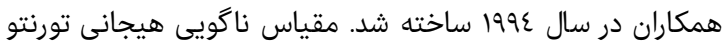

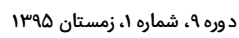

يرسشنامه سازگارى زناشويى لاك- والاس: اين يرسشنامه بر يايه

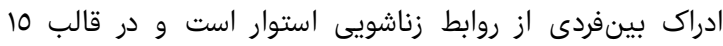

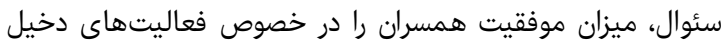

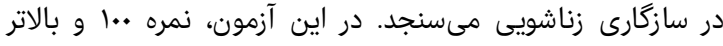

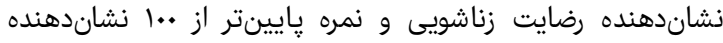

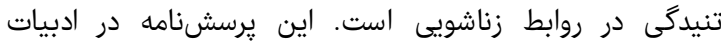

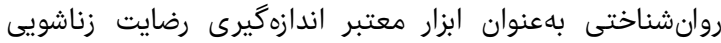

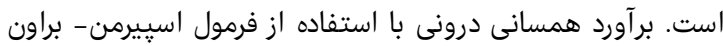

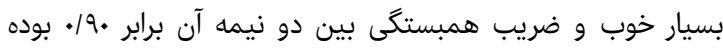

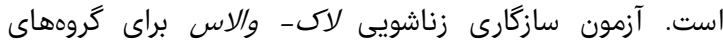

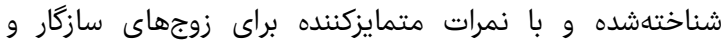

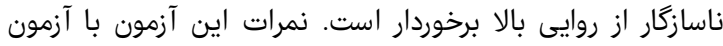

فصل نامه علمى - يزووهشى طب جانباز 


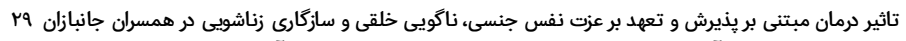

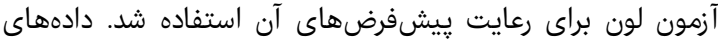

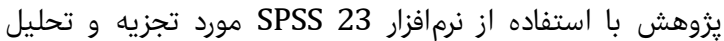

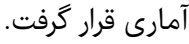

\section{يافتهها}

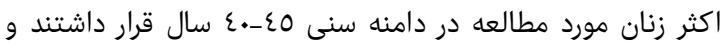

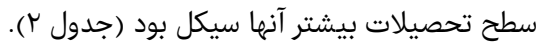

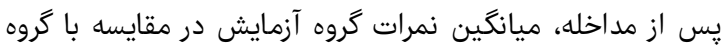

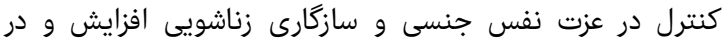

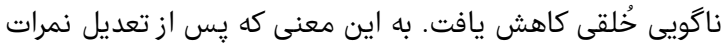

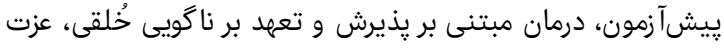

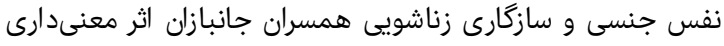

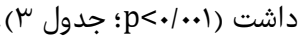

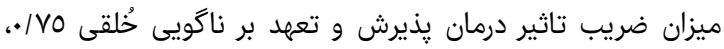

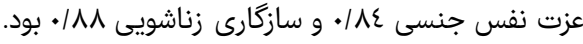

جدول س) مقايسه ميانكين نمرات ناكويى خلقى، عزت نفس جنسى و سازگًارى

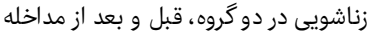

\begin{tabular}{|c|c|c|}
\hline 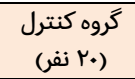 & 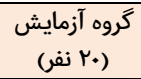 & شاخصها \\
\hline T./イ $\pm 7 / \varepsilon 0$ & $0 q / \varepsilon 0 \pm 0 / \mu\rceil$ & نيشاًّيى خُلقى \\
\hline $0 \wedge / V \varepsilon \pm 0 / I V$ & $\varepsilon \Lambda / \mu\urcorner \pm \mu / \Lambda r$ & يس آزمون \\
\hline & & عزت نفس جنسى \\
\hline$q \mu / \mu] \pm 7 / 70$ & $9 \varepsilon / 00 \pm V / 1 \mathrm{r}$ & 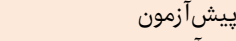 \\
\hline$q \vee / \mu O \pm V / \backslash \Lambda$ & $111 / / \Lambda \pm 11 / / r$ & ِِس آزمون \\
\hline & & سازگارى زناشويى \\
\hline 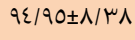 & $9 r / 7 \mu \pm V / / r$ & بيششآزمون \\
\hline 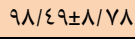 & $1.9 / \varepsilon V \pm 1 . / \varepsilon 0$ & ل عِس آزمون \\
\hline
\end{tabular}

بحث

هدف از يزوهش حاضر، بررسى تاثير درمان مبتنى بر يذيرش و

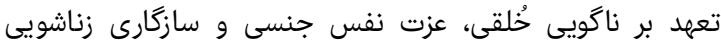

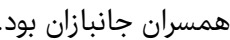

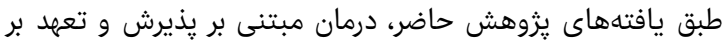

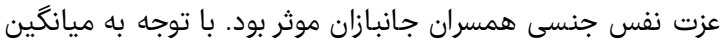

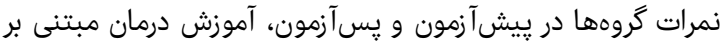

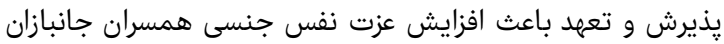

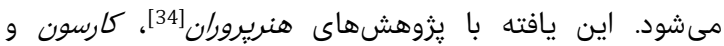

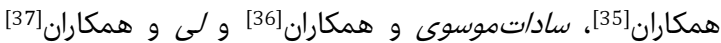
همخوانى دارد. در تبيين اين نتيجه مىتوان گفت دارد درمان مبتنى بر يذيرش و تعهد

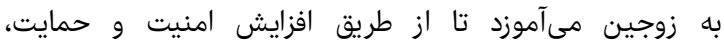

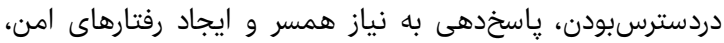

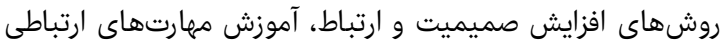

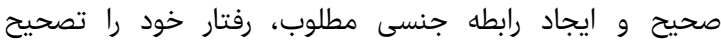

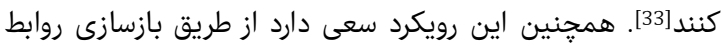

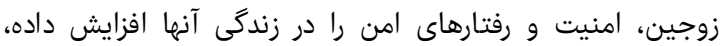

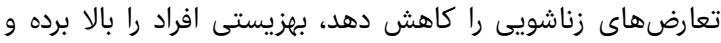

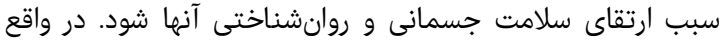

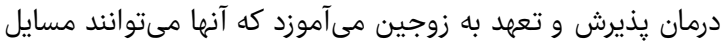

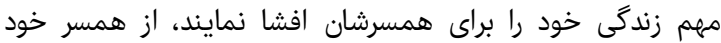

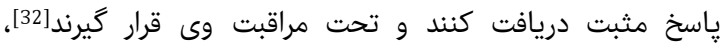
بهعلاوه تعاملهاى كلامى و غيركلامى خود را افزايش دون دهند
يك آزمون •rاسئوالى است و سه زيرمقياس دشوارى در شناسايى

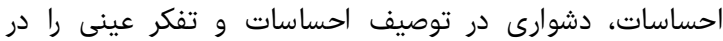
اندازههاى ينجدرجهاى ليكرت از نمره يك (كاملاً مخالف) تا نمره

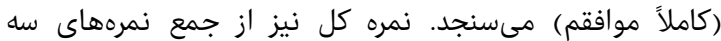

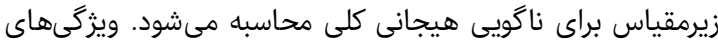

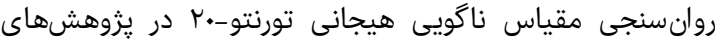

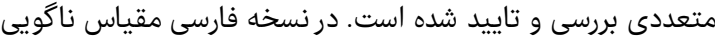

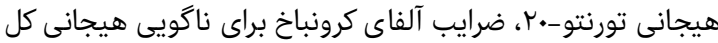

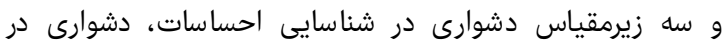

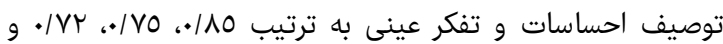

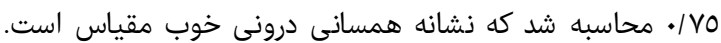

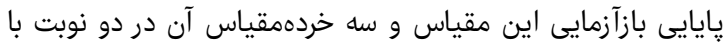

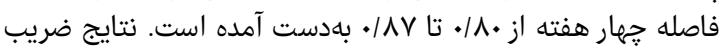

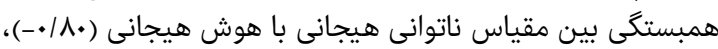

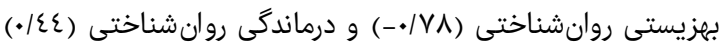
بيانگر روايى همزمان اين مقياس استى (40])

\begin{tabular}{|c|c|c|}
\hline 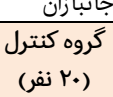 & 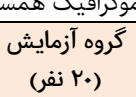 & متغيرهاى دموكرافيك \\
\hline $\begin{array}{c}\cdot \\
(\mu \cdot / \cdot)^{7} \\
\left.(7 \cdot / \cdot)\right|^{r} \\
(1 \cdot / \cdot) r\end{array}$ & $\begin{array}{l}(1 \cdot / \cdot) r \\
(\varepsilon \cdot / \cdot) \wedge \\
(\varepsilon 0 / \cdot) q \\
(0 / \cdot))\end{array}$ & 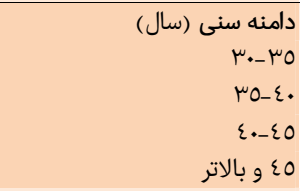 \\
\hline $\begin{array}{c}(0.1 \cdot)) . \\
(\mu 0 / \cdot) V \\
(10 / \cdot)^{\mu} \\
.\end{array}$ & $\begin{array}{l}(\varepsilon \cdot / \cdot) \wedge \\
(r 0 / \cdot) 0 \\
(r \cdot / \cdot) \varepsilon \\
(10 / \cdot) r\end{array}$ & 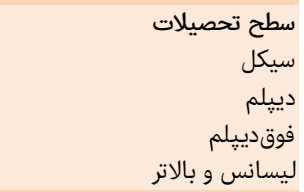 \\
\hline $\begin{array}{l}(\mathrm{rO} / \cdot) 0 \\
(0 . / \cdot)) \\
(\mathrm{YO} / \cdot) 0\end{array}$ & $\begin{array}{l}(\varepsilon \cdot / \cdot) \wedge \\
(\varepsilon 0 / \cdot)^{q} \\
(10 / \cdot)^{\mu}\end{array}$ & 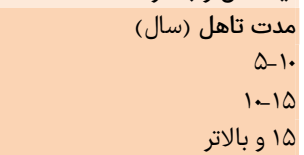 \\
\hline $\begin{array}{l}(\varepsilon 0 / \cdot)^{q} \\
(\varepsilon 0 / \cdot)^{q} q \\
(1 . / \cdot) r\end{array}$ & $\begin{array}{l}(\varepsilon \cdot 1 \cdot) \wedge \\
(0 . / \cdot)) . \\
(1 . / \cdot) r\end{array}$ & تعدفر تعرز فرزندان \\
\hline
\end{tabular}

آموزش يذيرش و تعهد در •ا جلسه •ودقيقهاى بهصورت هفتهاى

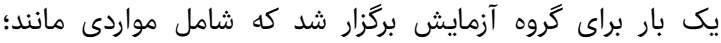

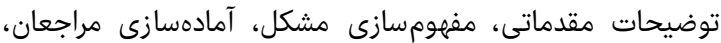

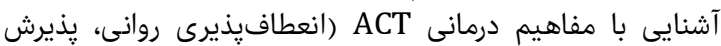

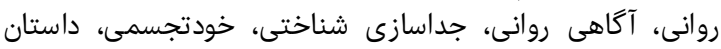

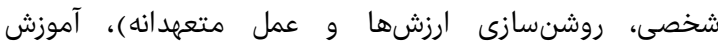

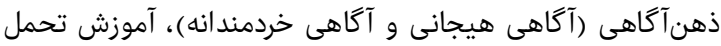

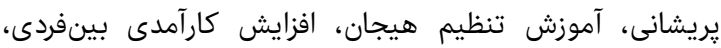

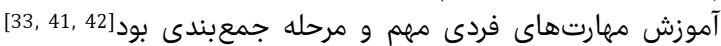

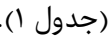

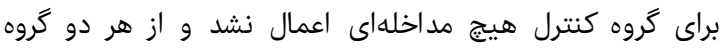

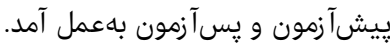

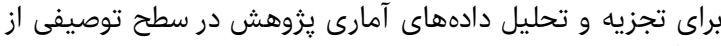

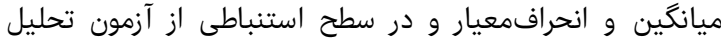

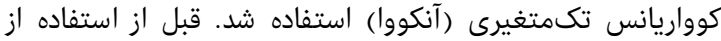

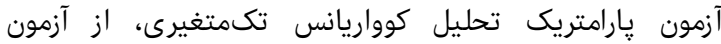

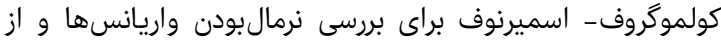

Volume 9, Issue 1, Winter 2017 
ايجاد مىشود نيست، بلكه بهمعنى عملكردهاى تازه نيز هست[45

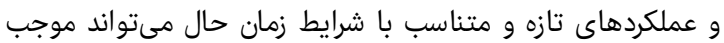

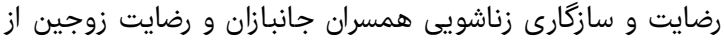

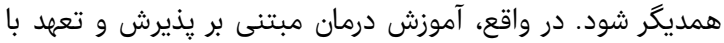

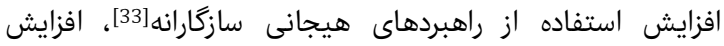

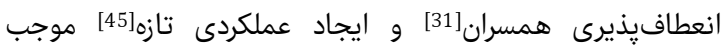
مى شود تا همسران به سطح بالاترى از سازگارى برسند.

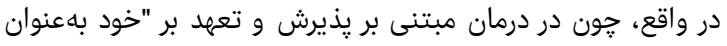

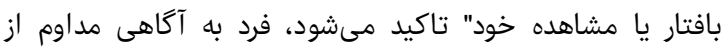

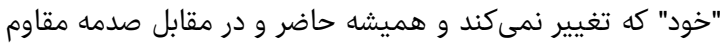

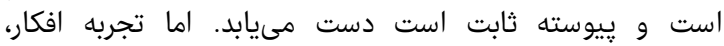

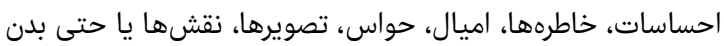

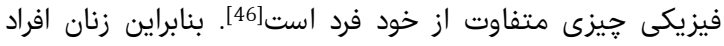

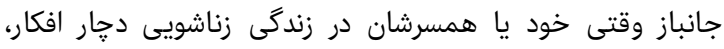

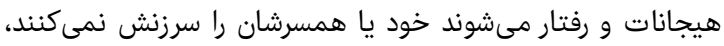

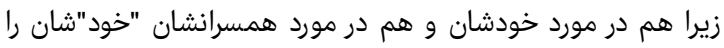

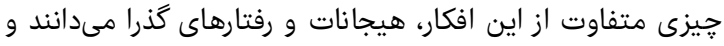

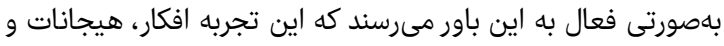

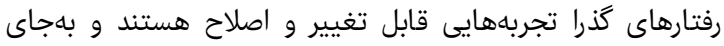

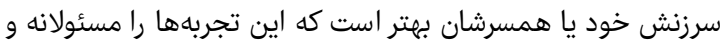

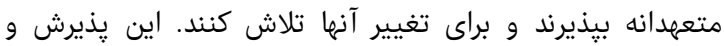

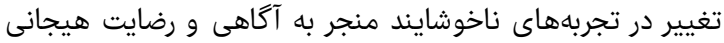

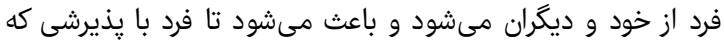

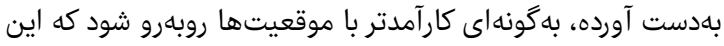

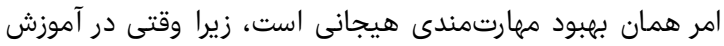

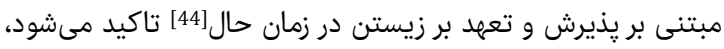

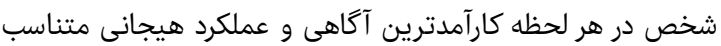
با آن لحظه را ابراز مى دند.

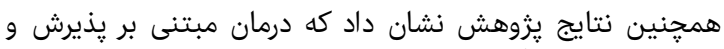

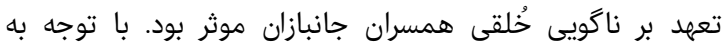

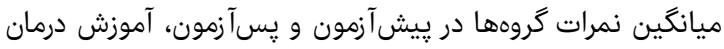

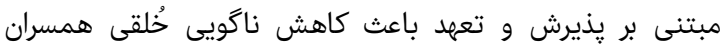

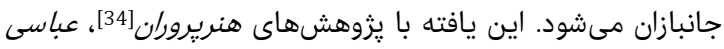

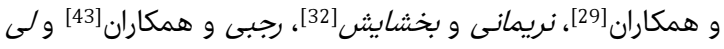

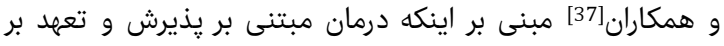
مشكلات هيجانى زوجين اثربخش است، همخوانى دارد.

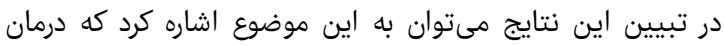

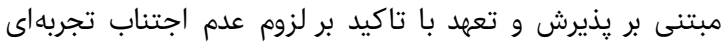

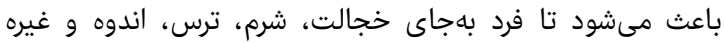

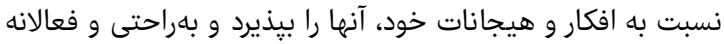

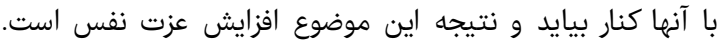

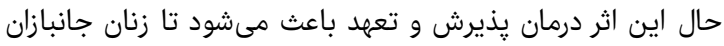

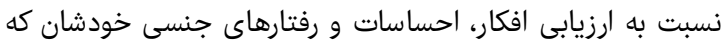

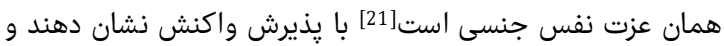

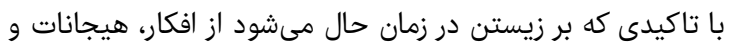

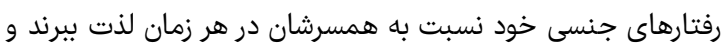

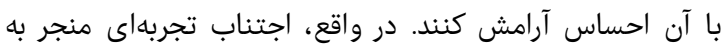

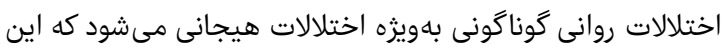

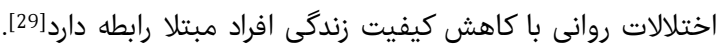

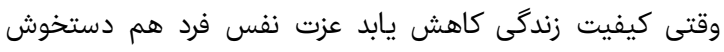

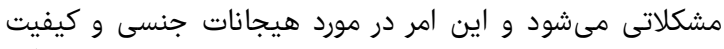

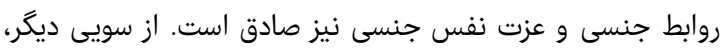

دوره ، شماره ا، زمستان هوس"

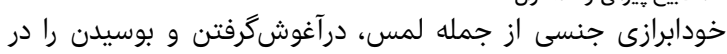

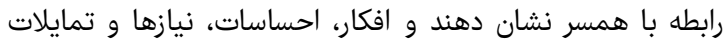

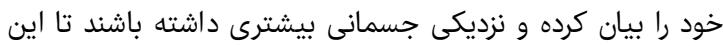

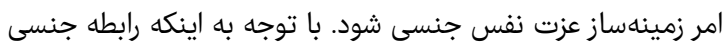

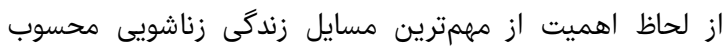

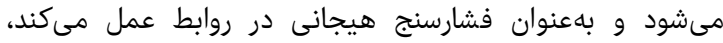

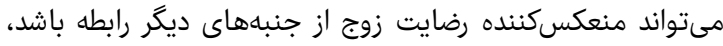

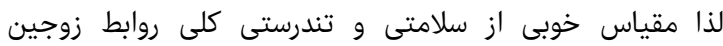

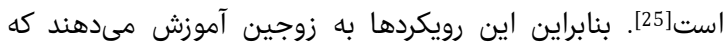

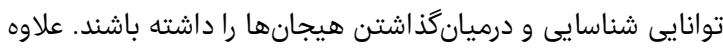

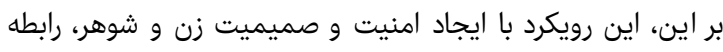
بين اين مهارتهاى هيجانى رويكرد بإيجاد امنيت وازگارى زناشويى را ميانجى مى مند [33]

همجنين نتايج پيزوهش نشان نشان داد كه درمان مبتنى بر يذيرش و

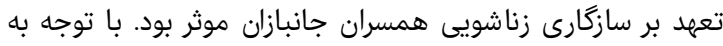

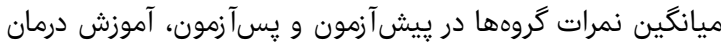

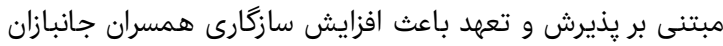

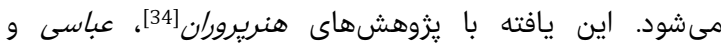

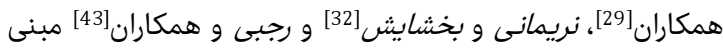

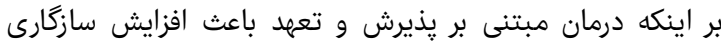
مى شود، همخوانى دارد.

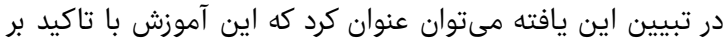

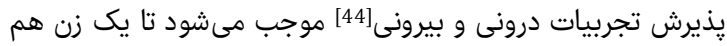

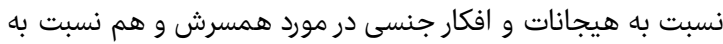

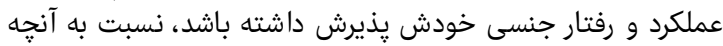

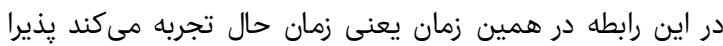

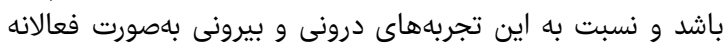

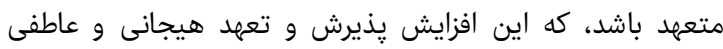

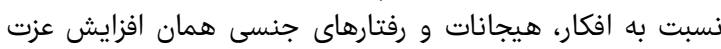

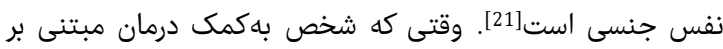

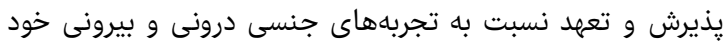

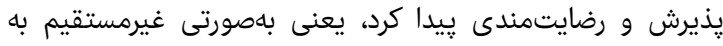

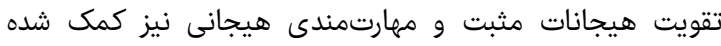
است، زيرا مهارتمندى هيجانى بهمعنى كاركرد هيجانى مانى ماهرانه دئ دريز

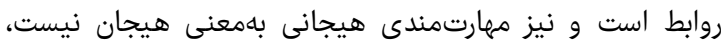

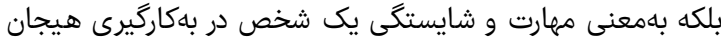

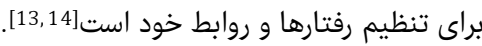

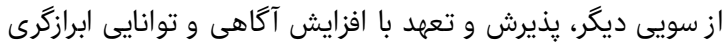

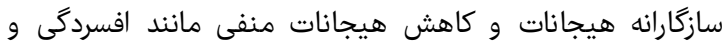

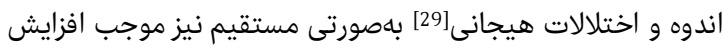

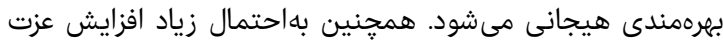

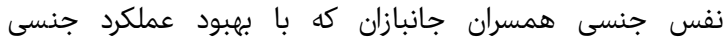

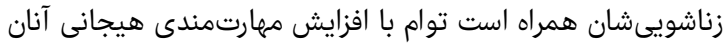

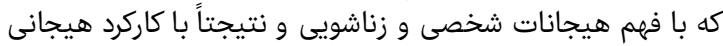

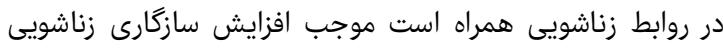

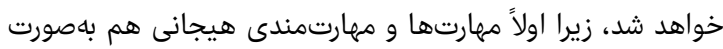

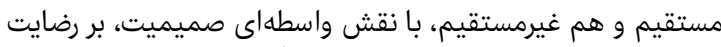

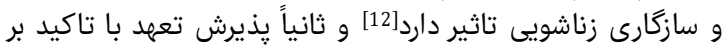

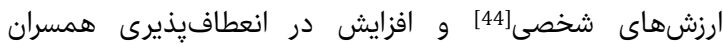

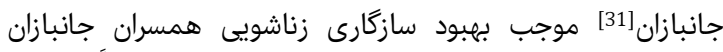

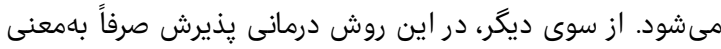

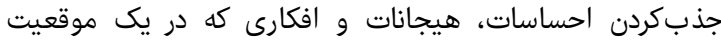




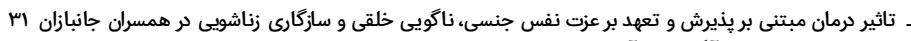

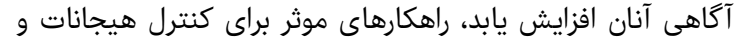

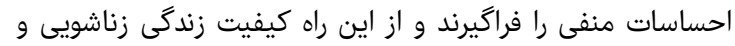

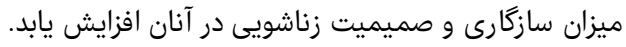

\section{نتيجهاگيرى}

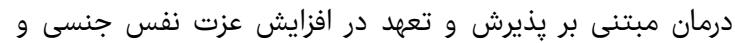

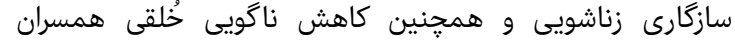
جانبازان موثر است.

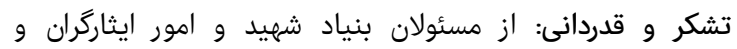

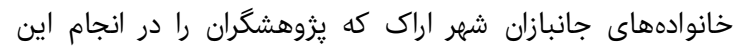

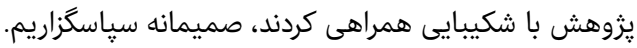

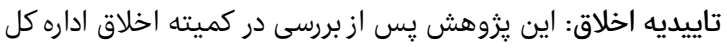

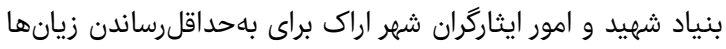

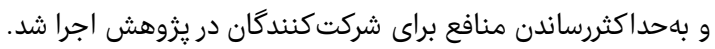

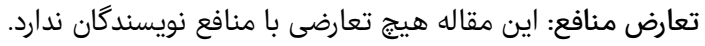

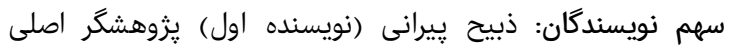

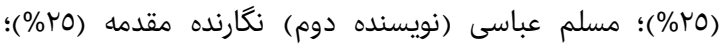

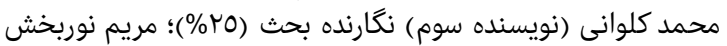

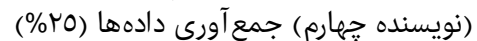

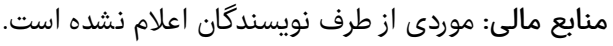

\section{منابع}

1- Mehdi-Araghy M. The effect of counseling on veteran's family. Proceeding of $1{ }^{\text {st }}$ Scientific Conference Affairs and Sport Veterans Affairs. Tehran: Iran Veterans Affairs; 2004. pp. 141-52. [Persian]

2- Dejkam M, Aminoroaya A. Comparing between psychological health of veterans 'wives and ordinaries' wives who had referred to Imam Hosein hospital and Sadr hospital. The 1st Scientific Conference of Veterans and Family. Tehran: Iran Veterans Affairs; 2003. [Persian] 3- Ghahari Sh, Mehryar AH, Birashk B. Comparitive study of some mental disorders in children of fatherless, martyred's, veteran's, and normal (7-12 years old) in Chalus city. J Mazandaran Univ Med Sci. 2003;13(41):8191. [Persian]

4- Afrooz Gh, Vysmeh A. The relationship between depression and how veterans sons with their fathers. J Psychol Educ Sci. 2001;31(2):35-50. [Persian]

5- Zerach G, Greene T, Solomon Z. Secondary traumatization and self-rated health among wives of former prisoners of war: The moderating role of marital adjustment. J Health Psychol. 2015;20(2):222-35.

6- Solomon Z, Debby-Aharon Sh, Zerach G, Horesh D. Marital adjustment parental functioning and emotional sharing in war veterans. J Fam Issues. 2011;32(1):127-47. 7- Faranak F, Ebrahiminejad GH, Razavi Nematolahi V. The relationship of body image and self-esteem with marital adjustment: a study on female employees of Kerman University of Medical Sciences. J Health Dev. 2014;3(4):304-12. [Persian]

8- Nazari A, Taherirad M, Asadi M. The Effectiveness of enhancement program on marital adjustment. J Fam Psychother. 2013;3(4):527-42. [Persian]

9- Peterson-Post KM, Rhoades GK, Stanley SM, Markman HJ. Perceived criticism and marital adjustment predict depressive symptoms in a community sample. Behav Ther. 2014;45(4):564-75.

10- Sinha SP, Mackerjee N. Marital adjustment and space orientation. J Soc Psychol. 1990;130(5):633-9.
درمان مبتنى بر يذيرش و تعهد در درمان يريشانى زوجها موثر [30]

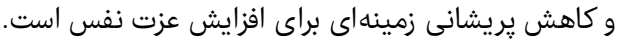

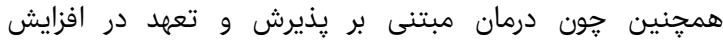

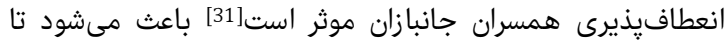

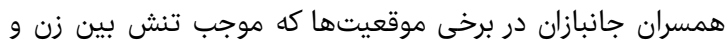

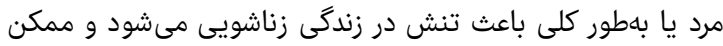

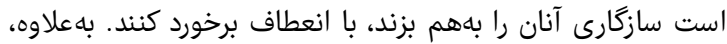

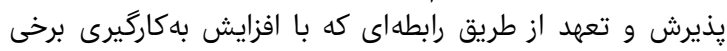

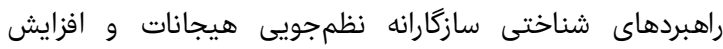

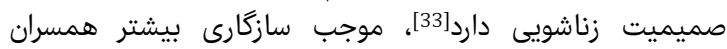

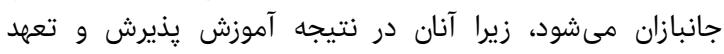

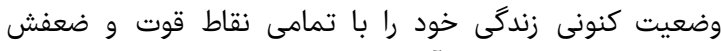

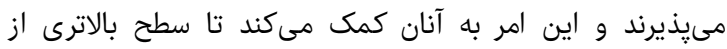
سازگًارى زناشويى را تجربه كنند.

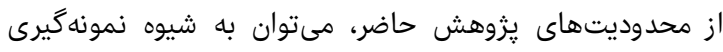

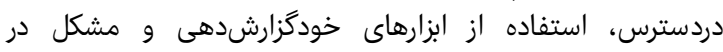

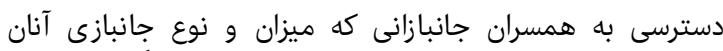

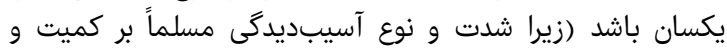

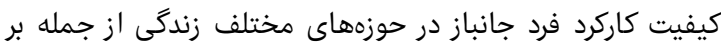

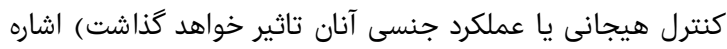

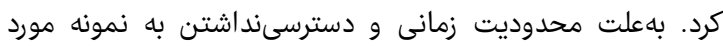

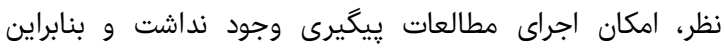

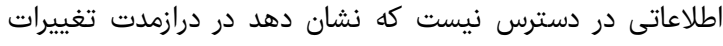

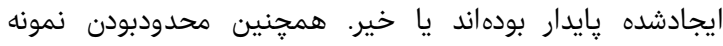

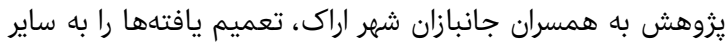

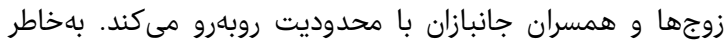

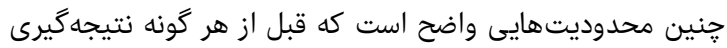

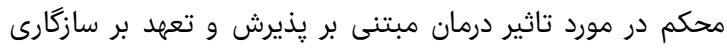

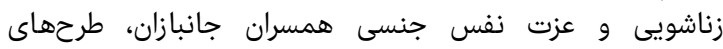
تحقيقاتى دقيق ديكر مورد نياز است.

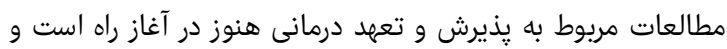

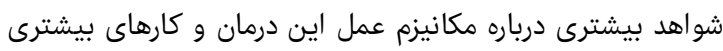

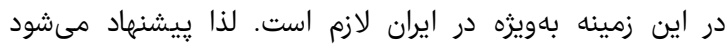

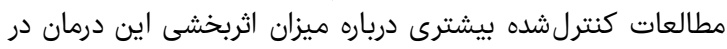

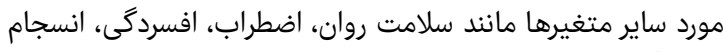

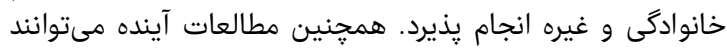

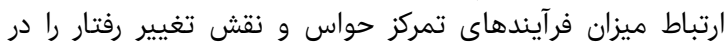

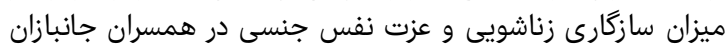

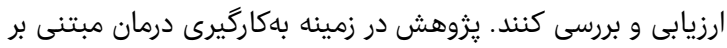

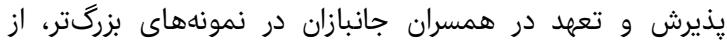

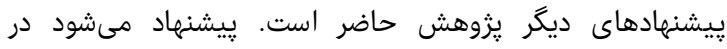

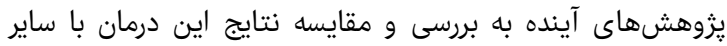

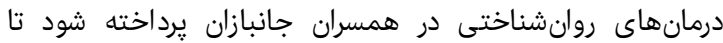

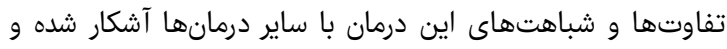

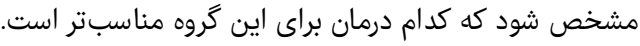

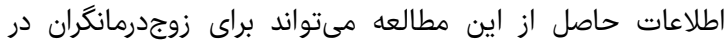

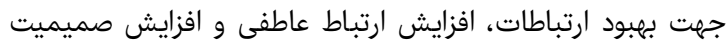

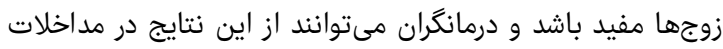

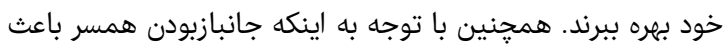

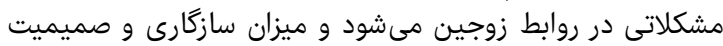

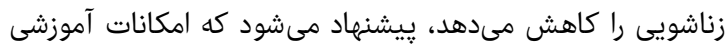
رايكان و كاركاههاى آموزشى براهي براى اين زوجها فراهم آيد تا سطح 
properties of persian version of acceptance and action questionnaire-II. J Psychol Model Method. 2013;3(10):65-80. [Persian]

30- Amanelahi A, Heydarianfar N, Khojastemehr R, Imani $\mathrm{M}$. The effectiveness of acceptance and commitment therapy (ACT) in couples's distress. J Appl Counsling. 2014;4(1):103-19. [Persian]

31- Moghtadayi M, Khosh Akhlagh H. Effectiveness of acceptance and commitment therapy on psychological flexibility of veterans' spouses. Iran J War Public Health. 2015;7(4):183-8. [Persian]

32- Narimani M, Bakhshayesh R. Effectiveness of acceptance and commitment based training on the psychological and emotional well-being, marital satisfaction in divorcing couples. J Counseling Res. 2015;13(52):108-23. [Persian]

33- Eslami E. The role of personal values, acceptance and cognitive emotional cognitive regulation-seeking in marital intimacy of married women [Dissertation]. Tehran: Kharazmi University; 2015. [Persian]

34- Honarparvaran $\mathrm{N}$. The Efficacy of acceptance and commitment therapy (ACT) of forgiveness and marital adjustment women damaged by martial infidelity. J Women Soc. 2014;5(3):135-50. [Persian]

35- Carson JW, Carson KM, Gil K, Baucom DH. Mindfulness-based relationship enhancement. Behav Ther. 2004;35(3):471-94.

36- Sadat Mousavi M, Rashidi A, Golmohammadian M. Effectiveness of acceptance and commitment therapy on increasing marital satisfaction of the veteran and freedman wives. J Appl Counseling. 2015;5(2):97-112. [Persian]

37- Lee V, Robin Cohen S, Edgar L, Laizner AM, Gagnon AJ. Meaning-making intervention during breast or colorectal cancer treatment improves self-esteem, optimism, and self-efficacy. Soc Sci Med. 2006;62(12):3133-45

38- Delavar A. Theoretical and practical research in the humanities and social sciences. Teharn: Roshd Press; 2011. [Persian]

39- Zeanah PD, Schwarz JC. Reliability and validity of the sexual self-esteem inventory women. Assessment. 1996;3(1):1-15.

40- Besharat A. Toronto alexithymia scale: Questionnaire, instruction and scoring (Persian version). Dev Psychol Iran Psychol. 2013;10(37):90-2. [Persian]

41- Forman EM, Herbert JD. New directions in cognitive behavior therapy: Acceptance based therapies. In: Fisher JE, editor. Cognitive behavior therapy: Applying empirically supported treatments in your practice. $2^{\text {nd }}$ Edition. Hoboken: Wiley; 2008. pp. 263-5.

42- Izadi R, Abedi M. Acceptance and commitment therapy. Tehran: Jangal Press; 2014. [Persian]

43- Rajabi Gh, Hayatbakhsh L, Taghipour M. Structural model of the relationship between emotional skillfulness, intimacy, marital adjustment and satisfaction. J Fam Couns Psychother. 2015;5(1):68-92. [Persian] 44- Hayes SC. Acceptance and commitment therapy, relational frame theory, and the third wave of behavioral and cognitive therapies. Behav Ther. 2004;35(4):639-65. 45- Ahmadi O. Comparison of experimental avoidance, cognitive and emotional failures in divorcing couples and normal couples of Shiraz city [Dissertation]. Ahvaz: Islamic Azad University; 2014. [Persian]

46- Hosseinaei A, Ahadi H, Fata L, Heidarei A, Mazaheri MM. Effect of group acceptance and commitment therapy (ACT)-based training on job stress and burnout. Iran J Psychiatry Clin Psychol. 2013;19(2):109-20. [Persian]
11- Tamaren KJ. Factors of emotional intelligence associated with marital satisfaction [Dissertation] Partial: Saint Louis University; 2010.

12- Dunham SM. Emotional skillfulness in African marriage: Intimate safety as a mediator of the relationship between emotional skillfulness and marital satisfaction [Dissertation]. Akron: Akron University. 2008. 13- Mirgain S, Cordova JV. Emotional skill and marital health: The association between observed and selfreported emotion skill, intimacy and marital satisfaction. J Soc Clin Psychol. 2007;26(9):941-67.

14- Cordova JV, Gee CB, Warren LZ. Emotional skillfulness in marriage; Intimacy as a mediator of the relationship between emotional skillfulness and marital satisfaction. J Soc Clin Psychol. 2005;24(2):218-35.

15- Olsen N. Emotion skills, problem-solving, and marital satisfaction: Investigation the mediating effect of emotion skills on the relationship between problem solving skills and marital satisfaction [Dissertation]. Worcester: Clark University; 2006.

16- King LA, Emmons RA, Woodley S. The structure of inhibition. J Res Personal. 1992;26(1):85-102.

17- Goldman RA, Greenberg LS. Promotion emotional expression and emotion regulation in couples. In: Douglas SK, Jeffery S, Hughes J, editors. Emotion regulation in couples and families: Pathways to dysfunction and health. Washington, DC: American Psychological Association; 2006. pp. 150-2.

18- Jafaryazdi H, Golzari M. Emotional intelligence and martial adjustment of married women employed in education. J Fam Res. 2005;1(4):379-92. [Persian]

19- Rafati F, Shafiei N, Sharif F, Ahmadi J. The effect of psychological status of students on their academic progress. J Shaheed Sadoughi Univ Med Sci. 2003;11(3):80-6. [Persian]

20 - Hoban Sh, Hoban G. Self-esteem, self-efficay and selfdirected learning: Attempting to undo the confusion. Int J Self Learn. 2004;1(2):7-25.

21- Beth AV, Julien B, Anthony FB, Michael CA. Psychopathy, sexual behavior and self-esteem, it's different forgirls. Person IndividDiffer. 2010;48(7):833-8. 22- Ramazani M, Dolatian M, Shams J, Alavi H. The correlation between self-esteem and sexual dysfunction and satisfaction in women. Arak Med Univ J. 2012;14(6):57-65. [Persian]

23- Showani E, Zahrakar K, Ghasemi Jobaneh R, Dargahi Sh. Role of sexual satisfaction, spiritual health, psychological distress and self- esteem in marital commitment of women's with veteran husbands. Iran J War Public Health. 2015;7(2):99-105. [Persian] 24- Pourfaraj Omran M. The effectiveness of acceptance and commitment group therapy in social phobia of students. Knowledge Health. 2011;6(2):1-5. [Persian] 25- Hayes SC, Strosahl KD. A practical guide to acceptance and commitment therapy. New York: Springer Science and Business Media Inc; 2010.

26- Zeidan F, Johnson SK, Diamond BJ, David Z, Goolkasian P. Mindfulness meditation improves cognition: Evidence of brief mental training. Conscious Cogn. 2010;19(2):597-605.

27- Bohlmeijer E, Prenger R, Taal E, Cuijpers P. The effects of mindfulness-based stress reduction therapy on mental health of adults with a chronic medical disease: A meta-analysis. J Psychosom Res. 2010;68(6):539-44.

28- Goldin PR, Gross JJ. Effects of mindfulness based stress reduction (MBSR) on emotion regulation in social anxiety disorder. Emotion. 2010;10(1):83-91.

29- Abasi E, Fti L, Molodi R, Zarabi H. Psychometric 